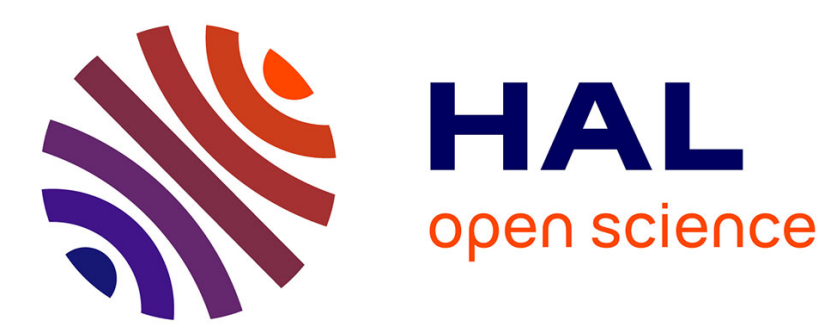

\title{
Acoustical and optical radiation pressures and the development of single beam acoustical tweezers
}

\author{
Jean-Louis Thomas, Régis Marchiano, Diego Baresch
}

\section{To cite this version:}

Jean-Louis Thomas, Régis Marchiano, Diego Baresch. Acoustical and optical radiation pressures and the development of single beam acoustical tweezers. Journal of Quantitative Spectroscopy and Radiative Transfer, 2017, 195, pp.55-65. 10.1016/j.jqsrt.2017.01.012 . hal-01438774

\section{HAL Id: hal-01438774 https://hal.science/hal-01438774}

Submitted on 18 Jan 2017

HAL is a multi-disciplinary open access archive for the deposit and dissemination of scientific research documents, whether they are published or not. The documents may come from teaching and research institutions in France or abroad, or from public or private research centers.
L'archive ouverte pluridisciplinaire HAL, est destinée au dépôt et à la diffusion de documents scientifiques de niveau recherche, publiés ou non, émanant des établissements d'enseignement et de recherche français ou étrangers, des laboratoires publics ou privés. 


\title{
Acoustical and optical radiation pressures and the development of single beam acoustical tweezers
}

\author{
Jean-Louis Thomas ${ }^{\mathrm{a}, *}$, Régis Marchiano ${ }^{\mathrm{b}}$, Diego Baresch ${ }^{\mathrm{a}, \mathrm{b}}$ \\ ${ }^{a}$ Sorbonne Universités, UPMC Univ Paris 06, CNRS UMR 7588, Institut des \\ NanoSciences de Paris, 4 place Jussieu, Paris, France \\ ${ }^{b}$ Sorbonne Universités, UPMC Univ Paris 06, CNRS UMR 7190, Institut Jean le Rond \\ d'Alembert, 4 place Jussieu, Paris, France
}

\begin{abstract}
Studies on radiation pressure in acoustics and optics have enriched one another and have a long common history. Acoustic radiation pressure is used for metrology, levitation, particle trapping and actuation. However, the dexterity and selectivity of single-beam optical tweezers are still to be matched with acoustical devices. Optical tweezers can trap, move and positioned micron size particles, biological samples or even atoms with subnanometer accuracy in three dimensions. One limitation of optical tweezers is the weak force that can be applied without thermal damage due to optical absorption. Acoustical tweezers overcome this limitation since the radiation pressure scales as the field intensity divided by the speed of propagation of the wave. However, the feasibility of single beam acoustical tweezers was demonstrated only recently. In this paper, we propose a historical review of the strong similarities but also the specificities of acoustical and optical radiation pressures, from the expression of the force to the development of single-beam acoustical tweezers.
\end{abstract}

Keywords:

Radiation pressure in optics, Radiation pressure in acoustics, Acoustical tweezers, Pseudo-momentum

1 1. Introduction

Radiation pressure is a mean force exerted by a wave that, in many situ-

ations, pushes an interface or a particle in the direction of propagation of the

\footnotetext{
${ }^{*}$ Corresponding author
} 
wave. This is usually interpreted as a transfer of linear momentum when the wave is scattered. Since the wave at linear order is seen as an oscillation with no mean momentum, radiation pressure is a nonlinear effect. It is common to track back the beginning of the long and complex history of radiation pressure to Kepler in 1619. On observing the specific shape of a comet's tail, he made the assumption that the radiation from the sun exerts a force on the comet's tail which changes its shape. The theoretical formulation of this hypothesis was made more than two centuries later by Maxwell, who introduced the stress tensor due to electromagnetic waves [1]. Hence, it is possible to compute the magnitude of the so-called radiation pressure exerted by the EM waves which is proportional to the ratio between the energy flux and the speed of the light. Therefore, the magnitude of this second order effect is very weak and the experimental demonstration of this force remained a real challenge for a quarter of a century. At the beginning of the twentieth century, two different experiments proposed by Lebedev [2], and Nichols and Hull [3] validated Maxwell's theory. On the acoustics side, the story began, as often, with the pioneering works of Lord Rayleigh [4, 5] who introduced the counterpart of the EM radiation pressure for acoustic waves. Shortly after Rayleigh's first paper and considering the experimental observations made by Dvorak on acoustic fountains [6], Altberg [7] proposed to use the radiation pressure to measure the amplitude of ultrasonic waves.

The experimental observation of the radiation pressure for electromagnetic and acoustic waves was a real challenge at this early stage. As mentioned above, radiation pressure is due to second order effect. But many phenomena may lead to second order effect and can contribute to an apparent force and to erroneous interpretation. In optics, Crooks' radiometer was claimed to be sensitive to radiation pressure. In fact, the force acting on Crooks' radiometer was due to temperature gradient and its magnitude was larger than the one expected. Nichols and Hull [3] improved the radiometer to avoid this effect and succeeded in measuring the radiation pressure with an amplitude in agreement with Maxwell's prediction. The situation is different in acoustics. Indeed, while non adiabatic behavior is common for gas, it is negligible for most liquids. However, wave attenuation occurs also due to viscosity. This is a transfer of a kind of linear momentum from the wave to the medium which generates a flow called acoustic streaming. This effect is small but grows with the propagation distance and time. It has to be distinguished from the radiation force, even though the distinction is not always simple [8]. Even though the energy is conserved, the situation 
remains subtle since the definition of mean linear momentum is quite difficult as soon as the wave propagates in a material medium. Indeed the momentum can be split in the wave momentum and the medium momentum and this is somewhat arbitrary. This leads to the controversy between Minkowsky and Abraham's momentum in optics. In acoustics, this also lead to many difficulties, and many papers are devoted to the concept of momentum and pseudo-momentum [9], [10]. Another difficulty, mostly in acoustics, comes from the different sources of nonlinearities (nonlinear terms appear both in the equation of momentum conservation and in the state equation). Combined with the notion of linear momentum for acoustic waves, this is at the core of the differences between Rayleigh and Langevin radiation pressure. Indeed, in acoustics Rayleigh and Langevin proposed two definitions of the radiation pressure due to a plane wave acting on an interface. This leads to different theoretical expressions of the radiation pressure, the Rayleigh's radiation pressure is sensitive to the nonlinear parameter of the medium, while Langevin's is not. Brillouin proposed a different approach which is not restricted to incident plane waves and he introduced a stress tensor which has many similarities with the Maxwell's stress tensor [11].

Radiation pressure offers the ability to apply forces without physical both in acoustics and in optics.

In acoustics, levitation traps have been known for a long time [12], [13], [14]. Levitation traps are generally based on standing waves. For particles which are very small in comparison with the wavelength, Gorkov proposed an elegant theory. He derived a theoretical formulation of the radiation pressure valid everywhere in an acoustic field due to standing waves. This formulation is widely used because many acoustics applications meet Gorkov's assumptions. For instance, acoustic radiation pressure is widely used in microfluidics to act on particles carried by the flow, it is called acoustophoresis $[15,16]$. Even if 3D manipulations are possible, these kinds of devices do not allow a selective control of a single particle $[17,18]$ because of the nature of the acoustic field. Indeed, standing waves possess a lot of nodes and maxima where the particles can be trapped in cluster. Despite its success and the increasing number of applications based on this approach, it is probably not the best one to develop selective traps, ie tweezers.

Solution was found in optics three decades ago by Ashkin who experimentally demonstrated the possibility to trap a single dielectric particle with a single-beam gradient force with a system called Optical Tweezers (OT in short). The first step which paves the way to the OT is the observation by 
Ashkin of the axial acceleration of particles illuminated by a laser beam and the presence of a transverse force which attracts the particles toward the beam axis [19]. This transverse force is now known as gradient force. The second step was proposed in the same paper [19]. In order to make an axial trap, Ashkin proposed to use a second laser sending a beam, whose propagation direction is opposed to the first one. Hence, the lateral forces are added while the axial ones subtract and do not engender an axial expelling. Particles are trapped. From this seminal work, the development of OT took sixteen years. In 1986, Ashkin et al. [20] proposed a new setup based on a sharply focused laser beam able to exert a negative pulling force on a particle located downstream from the focus. Hence, he demonstrated that a stable equilibrium position exists and that it is possible to trap a single dielectric particle with a single-beam gradient force. The negative force is due to a subtle effect of the back scattering field on the particle involving the physical properties of the particle and the incoming beam of light. The dexterity and selectivity of optical tweezers is significantly superior to others optical traps schemes and most applications of optical radiation pressure are made with optical tweezers.

In acoustics, soon after, $\mathrm{Du}$ and $\mathrm{Wu}$ suggested theoretically to use ultrasonic beams to trap and manipulate small elastic particles[21]. However, their analysis derived from Gorkov's theory confirmed that every solid elastic particle was expelled from the intensity maxima by the gradient force. Surprisingly in Wu's forthcoming experiment, the axial trapping failure was explained by the only presence of acoustic streaming [22]. Using a counterpropagating wave this axial expelling was canceled and trapping was obtained. This set-up is the acoustic equivalent of "all-optical light trap" [23]. More recently, two dimensional manipulation was achieved with a focused wave when the axial expelling is stopped by a membrane[24]. In optics, this scheme is coined "single beam traps" [23], [19]. In a series of papers $[25,26,27]$, we published the theory and the experimental observations of the first acoustical tweezers. The key was the shape of the beam. Indeed, previous studies used plane waves, gaussian beams or focused beams which exert a pushing force on any solid particles. After a careful analysis of the scattering problem [25], we proposed to use a singular beam, namely an acoustical vortex, which is a beam with a zero amplitude on its center [26, 27]. Finally, the experimental demonstration of the all-acoustical single beam tweezers was achieved by combining all these elements [27]. Figure 1 illustrates the concept of acoustical tweezers acting on a single elastic sphere. At the same 


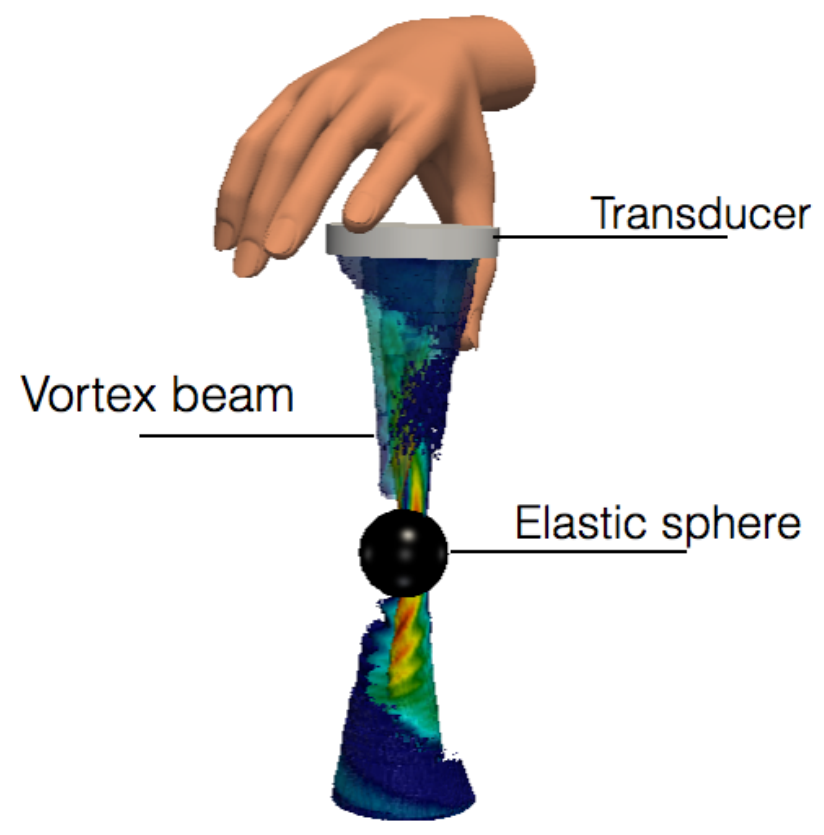

Figure 1: Schematic illustration of acoustical tweezers acting on a single elastic sphere. Note that, the size of the sphere is overstated to be visible

time another experimental demonstration was published [28]. The scheme are very similar, based on the same previous results, used the same kind of fields and the same kind of particles, polystyrene spheres. The main difference is that, the trapping is carried in air. Thus the buoyancy force is quite weak and the weight of the particle precludes any direct demonstration of the axial pulling force.

The goal of this paper is to give the key concepts which make optical and acoustical tweezers fascinating devices able to control one particle remotely. Therefore, the radiation pressure due to EM or acoustical waves is presented in section 1. Special attention is paid to the derivation of Maxwell's stress tensor and its analogous Brillouin's stress tensor. These two tensors show that even though the radiation pressure is a nonlinear effect, it can be computed from the first order wave fields both in optics and acoustics. Therefore, solving the canonical problem of a sphere illuminated by an arbitrary shaped incident beam is mandatory. This is done thanks to the Generalized Lorenz- 
Mie Theory in optics and its counterpart in acoustics, which are discussed in section 3. The GLMT provides a complete framework allowing to express the radiation pressure exerted on a sphere from the physical properties of the sphere and the parameter of the beam. The special case of small particles compared to the wavelength is discussed for acoustic waves in order to make bridges with usual formulations (Gorkov theory). Section 4 is devoted to the comparison of optical and acoustical tweezers. In particular, the choices associated to the properties of elastic particles and the incident beam are discussed in details for the acoustical case. Finally, a presentation of the advantages and the drawbacks of the two kinds of tweezers is proposed.

\section{Radiation pressure and stress tensor}

In this section, we review and compare the mathematical expression for radiation pressure in optics and in acoustics. We emphasize the strong similarities of the final expressions even though the derivation is different to accommodate the specificity of each field. At the end, we compare the optical and acoustical radiation pressures exerted by a plane wave and their relations with pseudo-momentum.

\subsection{Radiation pressure tensor for an optical field}

The first step consists in writing the equation of conservation of mechanical momentum and taking the average in time. The change in momentum of free charges, $\mathbf{P}_{\text {mech }}$, is related to the forces applied, i.e the Lorentz force:

$$
\frac{\partial \mathbf{P}_{m e c h}}{\partial t}=\int_{V}(\rho \mathbf{E}+\mathbf{J} \wedge \mathbf{B}) d V
$$

First we consider a homogeneous medium : $\nabla \epsilon_{i j}^{r}=0$ and $\nabla \mu_{i j}^{r}=0$, where $\epsilon^{r}$ and $\mu^{r}$ are respectively the relative permitivity and the relative permeability. The latin subscripts $(i, j)$ are used for spatial coordinates. From this equation and using Maxwell equations for a homogeneous medium without any electrostriction or magnetostriction, one can easily derive a continuity equation [29]:

$$
\frac{\partial}{\partial t}\left(\mathbf{P}_{m e c h}+\int_{V} \mathbf{G}_{M} d V\right)+\int_{V} \nabla \cdot(-\mathcal{M}) d V=0
$$


with

$$
\begin{gathered}
\mathcal{M}_{i, j}=E_{i} D_{j}+B_{i} H_{j}-1 / 2\left(E_{k} D_{k}+B_{k} H_{k}\right) \delta_{i j} \\
\mathbf{G}_{M} \quad=\mathbf{D} \wedge \mathbf{B}=\mathbf{E} \wedge \mathbf{H} / c_{n}^{2}=\mathbf{S}_{o} / c_{n}^{2}
\end{gathered}
$$

In these equations, $c$ is the speed of light in vacuum, $c_{n}$ the phase velocity of light in the medium of index $n . \mathbf{G}_{M}$ is known as the Minkowski pseudomomentum, $\mathcal{M}$ is the Maxwell stress tensor and $\mathbf{S}_{o}$ is the Poynting vector. If the medium considered contains no free charge carrier, $\mathbf{P}_{\text {mech }}=0$, we obtain a continuity equation for the Minkowsky pseudo-momentum, $\mathbf{G}_{M}$ [9], and $-\mathcal{M}$ is its flux. Note that the derived continuity equation, Eq. 2, is obtained with the assumption of a homogeneous medium. The general equation contains two terms proportional to $\nabla \epsilon_{i j}^{r} 0$ and $\nabla \mu_{i j}^{r}$ and is not a continuity equation. Hence Eq. 3 is based on the homogeneity of the medium, i.e invariance with respect to a spatial translation of the material medium. This remark emphasizes the difference with the true linear momentum conservation. Indeed, the linear momentum is related to the invariance with respect to translation of spatial coordinates [9].

The radiation pressure is a mean force, the next step is to take the time average, noted \langle\rangle , of Eq. 3 for a medium with no free charge. Note that for any stationary of periodic field, the mean of the derivative in time is equal to zero. This yieds:

$$
\nabla \cdot\langle\mathcal{M}\rangle=0
$$

When there is an interface, for instance for a dielectric particle $\nabla \epsilon_{i j}^{r} \neq 0$, the conservation of $\langle\mathcal{M}\rangle$ is no longer true and a force is applied. This force is equal to the integral of the stress tensor on the surface of the particle, $\Sigma$ :

$$
\mathbf{F}=\int_{S}\langle\mathcal{M}\rangle \cdot \mathbf{n} d S
$$

where $\mathbf{n}$ is the unit vector normal to the surface element of the particle and pointing outward.

However using the conservation of the flux of pseudo-momentum, Eq. 5, and the theorem of divergence, the integral can be performed on any closed surface, $S_{R}$, outside the particle.

$$
\mathbf{F}=\int_{S}\langle\mathcal{M}\rangle \cdot \mathbf{n} d S=\int_{S_{R}}\langle\mathcal{M}\rangle \cdot \mathbf{n}_{R} d S_{R}
$$


One last remark, the definition of $\mathcal{M}_{i, j}$, Eq. 3, shows that all terms are quadratic quantities. This yields that this tensor can be computed at order 2 with the linear fields.

\subsection{Radiation pressure tensor for an acoustical field}

In optics we have obtained a stress tensor, the Maxwell stress tensor, and identified the radiation pressure as the average of the stress applied on the particle surface. The radiation pressure is of second order since all terms appearing in the expression of the Maxwell stress tensor are quadratic with respect to the electric and magnetic fields. In acoustics, the stress tensor is well identified and, the force on the object is by definition the integral of the stress tensor on the surface of the particle. For a fluid the stress tensor $\sigma_{i j}$ reduces to the pressure $-P \delta_{i j}$. However an acoustic wave is a displacement of the material particles of the medium of propagation. Therefore the surface of the object is moving and the amplitude of this displacement is proportional to the acoustic field. We can conclude that, as in the optical case, this quantity is at least of second order:

$$
\mathbf{F}=-\int_{S(t)} P \mathbf{n} d S
$$

and the radiation pressure is the mean component of this force

$$
\langle\mathbf{F}\rangle=-\left\langle\int_{S(t)} P \mathbf{n} d S\right\rangle
$$

To get an expression more tractable and make an analogy with optical radiation pressure, the fixed Euler coordinates are more convenient. The two points of view, Lagrange and Euler coordinates, are equivalent [30]. The tensorial theory of radiation pressure in Euler coordinates was established by Brillouin in a series of paper and an account of this contribution and these references can be found in his text book, [11]. As in optics, the first step is to write the continuity equation for momentum:

$$
\begin{array}{r}
\frac{\partial \rho \mathbf{v}}{\partial t}+\nabla \cdot \mathcal{B}=0 \\
\mathcal{B}_{i j}=\rho v_{i} v_{j}+P \delta_{i j}
\end{array}
$$

where $\rho$ is the specific mass and $\mathbf{v}$ the particle velocity. This equation can be integrated in a volume bounded on one side by the vibrating surface of the 
particle and on the other side by a fixed surface in the fluid medium, Fig. 2.2. Using the divergence theorem, we get:

$$
\int_{V(t)} \frac{\partial \rho \mathbf{v}}{\partial t} d V+\int_{S(t)} \mathcal{B} \cdot \mathbf{n}^{\prime} d S+\int_{S_{R}} \mathcal{B} \cdot \mathbf{n}_{R} d S_{R}=0
$$

$\mathbf{n}^{\prime}$ is outward-pointing with respect to $V(t)$ and hence is the opposite of $\mathbf{n}$ as defined in Eq. 9. The first term would cancel with time averaging if we could commute the integral volume and the derivative in time. This mathematical step is the Reynolds transport theorem:

$$
\frac{\partial}{\partial t} \int_{V(t)} \rho \mathbf{v} d V=\int_{V(t)} \frac{\partial \rho \mathbf{v}}{\partial t} d V+\int_{S(t)} \rho \mathbf{v}\left(\mathbf{v} \cdot \mathbf{n}^{\prime} d S\right)
$$

Using this theorem, the continuity relation can be rewritten:

$$
\frac{\partial}{\partial t} \int_{V(t)} \rho \mathbf{v} d V+\int_{S(t)} P \mathbf{n}^{\prime} d S+\int_{S_{R}} \mathcal{B} \cdot \mathbf{n}_{R} d S_{R}=0
$$

We can now take the average in time and as in the optical case use the fact that the mean of a time derivative cancels:

$$
\mathbf{F}=-\left\langle\int_{S(t)} P \mathbf{n} d S\right\rangle=-\int_{S_{R}}\langle\mathcal{B}\rangle \cdot \mathbf{n}_{R} d S_{R}
$$

This expression is already comparable to the optical case, Eq. 7, the minus sign comes from the tensor used in acoustics, the flux of momentum, rather than a stress tensor. The radiation pressure is the integral of the flux of momentum on a closed surface that delineates a volume containing the particle. To get this we needed to take into account the first specificity of acoustics : the surface of the object is vibrating due to the presence of the acoustic field. Since the radiation pressure is a second order effect, this vibration, while of weak amplitude, is not negligible. The second specificity is that we don't directly get a quadratic expression of the linear fields. To proceed further, we need to perform a perturbative decomposition of the fields up to second order. Assuming no flow at rest, this yields at second order:

$$
\begin{array}{rc}
\mathbf{v}= & \mathbf{v}^{1}+\mathbf{v}^{2} \\
P= & P^{0}+P^{1}+P^{2} \\
\rho & =\rho^{0}+\rho^{1}+\rho^{2} \\
c_{a} & =c^{0}+c^{1}+c^{2}
\end{array}
$$




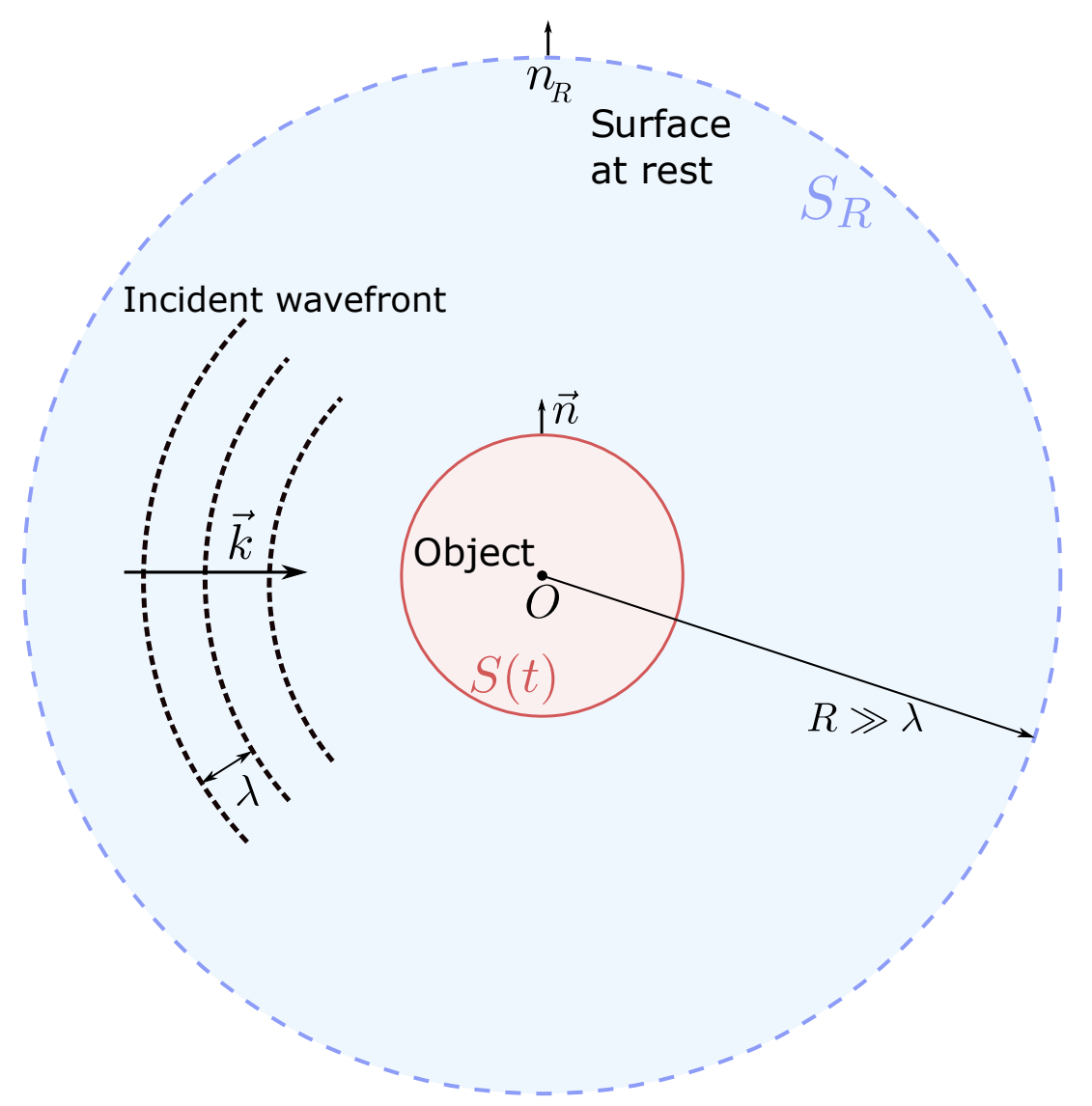

Figure 2: Scattering by a sphere. 
where $c_{a}$ is the speed of sound in the medium.

Many papers have been written to derive the equation giving the pressure at second order. This stage must be done very carefully and the boundary conditions must be taken into account [31], [32],[33]. For instance for a plane wave, i.e with an infinite lateral extension, or for a fluid which is laterally constrained by rigid wall, we get an expression that involves the non linearity of the state equation of the medium. This is the case studied by Rayleigh. However for practical cases, the radiation pressure is computed for an object embedded in a fluid or the wave is of limited extension. Contrary to the Rayleigh radiation pressure, here a static strain can relax laterally. This is the case presented in this review and the expression for the pressure at second order does not involve the nonlinear coefficient of the medium of propagation. These differences were first studied by Langevin and his analysis published by Biquard $[34,35]$.

$$
P_{E}^{2}=\frac{1}{2}\left(\frac{1}{\rho^{0}}\left(\frac{P^{1}}{c^{0}}\right)^{2}-\rho^{0}\left(\vec{v}^{1}\right)^{2}\right)
$$

$$
\mathcal{B}_{i j}=\rho^{0} v_{i}^{1} v_{j}^{1}+\frac{1}{2}\left(\frac{1}{\rho^{0}}\left(\frac{P^{1}}{c^{0}}\right)^{2}-\rho^{0} v_{k}^{1} v_{k}^{1}\right) \delta_{i j}
$$

As for Maxwell's tensor, there is an isotropic term analog to a pressure and a tensorial term. However, the isotropic term is not the energy per unit volume yet. To ease the comparison with the optical case this last expression can be rewritten:

$$
\mathcal{B}_{i j}=\frac{1}{2 \rho^{0}}\left(\frac{P^{1}}{c^{0}}\right)^{2} \delta_{i j}+\rho^{0} v_{i}^{1} v_{j}^{1}-\frac{1}{2}\left(\frac{1}{\rho^{0}}\left(\frac{P^{1}}{c^{0}}\right)^{2}+\rho^{0} v_{k}^{1} v_{k}^{1}\right) \delta_{i j}
$$

The two tensors $\mathcal{B}$ and $\mathcal{M}$ can now be compared. Both involve two fields $\mathbf{E}, \mathbf{H}$ and $p, \mathbf{v}$ respectively. The tensors split into an isotropic part and a tensorial part. The isotropic part is the energy density. The sum of the potential and kinetic energy in acoustics and the sum of the electric and magnetic energy in electromagnetism. These two energies yield two tensorial terms in optics. Acoustics gives an analog result but for a fluid medium the pressure field is a scalar. Note that the coefficient $1 /\left(\rho c_{0}^{2}\right)$ is the bulk compressibility of the medium. However, one difference remains. For the optical case, we get the conservation of the pseudo-momentum while in acoustics we used 
the conservation of momentum. We will see below that in the case studied here corresponding to the case derived by Langevin, this is also a pseudomomentum. Here, we would like to emphasize one more time and after many papers on this subject that the momentum on an acoustic wave is null.

\subsection{The acoustic momentum and pseudo-momentum}

An acoustic wave is an oscillation of particles and no net flux is expected. Indeed, the momentum is

$$
\rho \mathbf{v}=\rho^{0} \mathbf{v}^{1}+\rho^{1} \mathbf{v}^{1}+\rho^{0} \mathbf{v}^{2}
$$

If we take the time average, we get:

$$
\langle\rho \mathbf{v}\rangle=\left\langle\rho^{1} \mathbf{v}^{1}\right\rangle+\rho^{0}\left\langle\mathbf{v}^{2}\right\rangle
$$

So the mean mass flux is null if : $\left\langle\rho^{1} \mathbf{v}^{1}\right\rangle=-\rho^{0}\left\langle\mathbf{v}^{2}\right\rangle$. It can be demonstrated that this is the case for a plane wave, [36]. Counter-intuitively, $\left\langle\mathbf{v}^{2}\right\rangle$ points toward the source of sound. This quantity is a Eulerian quantity and represents a physical quantity in a fixed position in space and not a quantity related to a given material particle. The mean velocity in Lagrangian coordinates, i.e. the mean velocity of material particles, is null as expected. $\left\langle\mathbf{v}^{2}\right\rangle$ appears when the change of coordinates is performed and is the opposite of the Stokes drift. Using the state equations at first order, $p^{1}=\left(c^{0}\right)^{2} \rho^{1}$, we get:

$$
\rho^{1} \mathbf{v}^{1}=P^{1} \mathbf{v}^{1} /\left(c^{0}\right)^{2}=\mathbf{S}_{a} /\left(c^{0}\right)^{2}
$$

So while the total momentum is null, there is a finite pseudo-momentum that can be written as a quadratic expression of linear fields. The pseudomomentum is equal to the acoustic Poynting vector, $\mathbf{S}_{a}$, divided by the square of the phase velocity in the medium. This is the relation obtained above with Minkowsky pseudo-momentum in the optical case, Eq. 4. In the next section we will see that the radiation pressure is related to this pseudo-momentum.

\subsection{Acoustical pseudo-momentum for a plane wave}

Let us consider a plane wave propagating along $z$ and hence $\mathbf{v}^{1}=\left(0,0, v^{1}\right)$. The flux of momentum is :

$$
\mathcal{B}=\rho^{0}\left(v^{1}\right)^{2} \delta_{z z}+\frac{1}{2}\left(\frac{1}{\rho^{0}}\left(\frac{P^{1}}{c^{0}}\right)^{2}-\rho_{0}\left(v^{1}\right)^{2}\right) \delta_{i j}
$$




$$
\langle\mathcal{B}\rangle \cdot \mathbf{z}=\langle E\rangle=\left\langle\frac{S_{a}}{c^{0}}\right\rangle=\left\langle c^{0} \rho^{1} v^{1}\right\rangle
$$

For this simple case, the radiation pressure is equal to the energy density, the Poynting vector divided by the phase velocity, or the pseudo-momentum times the phase velocity.

\subsection{Optical pseudo-momentum for a plane wave}

We consider a plane wave propagation along $\mathbf{z}$ and hence $\mathbf{E}=(E, 0,0)$ and $\mathbf{B}=(0, B, 0)$. The Maxwell stress tensor is:

$$
\mathcal{M}=\epsilon E^{2} \delta_{x x}+\frac{1}{\mu} B^{2} \delta_{y}-\frac{1}{2}\left(\epsilon E^{2}+\frac{B^{2}}{\mu}\right) \delta_{i j}
$$

The component of the flux of pseudo-momentum along the direction of propagation, $\mathbf{z}$ is :

$$
-\langle\mathcal{M}\rangle \cdot \mathbf{z}=\langle E\rangle=\left\langle\frac{S_{o}}{c_{n}}\right\rangle=\left\langle c_{n} G_{M}\right\rangle
$$

The optical case is identical to the acoustical case but the mean of the Minkowski pseudo-momentum replaces the mean of the acoustic pseudomomentum, $\rho_{1} v_{1}$.

\section{Radiation force on a sphere exerted by an arbitrary shaped in- cident beam}

As long as the free-field condition is fulfilled, Langevin's expression of the excess of pressure can be used and the force is computed using Brillouin's stress or pseudo-momentum tensor. The latter only involves quadratic expressions of the first order field as recalled in the previous section. Hence, one needs to calculate the total linear field (the sum of incident and reflected waves) and then to compute the force from these quantities. In this paper we pay attention interaction of waves with spheres as it is a very important case. First of all, the Lorenz-Mie theory is presented. This theory holds for incident plane waves on a sphere. When the incident beam is not plane, different strategies have been developed. Here we focus on the Generalized Lorenz-Mie theory and in particular its extension to acoustics radiation problems which permits to compute the scattered field for an arbitrary beam. Then, considerations on the beam shape coefficients is proposed. Finally, the regime for which the size of the particle is very small compared to the wavelength is studied both in acoustics and optics. 


\subsection{Scattering by a plane wave by a sphere}

The first derivation of the scattering of a plane wave by an elastic sphere in an inviscid fluid was developed by Faran [37]. Interested in the propagation of ultrasound through suspensions and emulsions like aerosols or other diphasic solutions an important contribution was made by Epstein and Carhart [38] that dealt with the scattering of a thermo-viscous fluid droplet suspended in a thermo-viscous fluid. Allegra and Hawley generalized this model to the case of a visco-elastic sphere [39]. This model is commonly refereed to as the ECAH model in acoustics. Importantly, an elastic medium supports the existence of a one compressional and two transverse shear waves. Mathematically the displacement vector can be decomposed into its irrotational and solenoidal parts using Helmholtz decomposition. The latter is the curl of a potential vector $\mathbf{A}$. As in electromagnetism, this decomposition allows some freedom and the vector potential $\mathbf{A}$ satisfies the gauge condition $\nabla \cdot \mathbf{A}=0$. An interesting analogy lies in that this acoustic potential $\mathbf{A}$ and both the electric and magnetic fields $\mathbf{E}, \mathbf{B}$ are solutions of the vector Helmholtz equation. Consequently, the same mathematical steps can be followed to retrieve the solution. Nevertheless, the early developments in acoustics all considered the restricted case of a plane longitudinal wave incident on a spherical scatterer $[37,38,39,40]$. Owed to this strong symmetry condition the vector potential in spherical coordinates $(r, \theta, \phi)$ can be written $\mathbf{A}=(0,0, A(r, \theta))$ for a plane wave propagating along the $z$ axis. The problem is thereafter considerably simplified inside the sphere and the scalar potential $A$ is solution of the scalar Helmholtz equation. It can be written as an infinite sum of spherical modes. The incident plane wave is expanded on the same spherical modes and applying the correct boundary conditions yields the expression of the unknown scattering coefficients necessary to calculate the total external field. One can thereafter evaluate the force exerted on a compressible sphere [41], [42].

It is noteworthy that a transversely polarized wave immediately breaks the aforementioned azimuthal symmetry. It is the case for the scattering of electromagnetic waves and the modern solution often referred to as the Mie or Lorenz-Mie theory can actually be tracked back to Clebsch and his solution for the scattering of elastic waves by a rigid sphere [43, 44]. There are various examples of the resolution of this type of boundary-value problem in acoustics e.g. [45], [46]. A computationally useful method based on the "TMatrix" [47] was initially introduced in acoustics by Waterman [48, 49]. This matrix does not depend on the nature of the incident wave. It is completely 
defined by the scatterer and the propagation medium.

\subsection{Scattering of an arbitrarily shaped beam by a sphere}

With the growing interest in contact-less particle manipulation, recent research has extended these approaches to the case of a particle located on the axis of an incident acoustic beam. Examples include axisymmetric beams [50, 51] or the more complex wavefronts of a helicoidal bessel beam [52, 53]. To deal with the scattering problem, the Bessel beam was decomposed as a series of plane waves and the use of the previously cited results are straightforward. Using an angular spectrum decomposition of the incident field, this result was generalized to the case of a beam with arbitrary wavefront and the radiation pressure was computed in three dimensions [54].

In optics, an important approach referred to as the Generalized LorenzMie Theory (GLMT) was developed [55, 56, 57], [58]. In the GLMT, the problem under consideration is the scattering of an arbitrary incident wave by an arbitrarily located dielectric particle. This model was successful in obtaining the radiation pressure exerted by a beam of arbitrary wavefront regardless on the relative position of the sphere [59], [60]. This efficient approach was recently adapted to acoustics [25]. The incident wave is decomposed in the spherical basis centered on the sphere using beam shape coefficients. Note that even for a compressional incident acoustic wave, considering an arbitrary wavefront or an arbitrary location of the particle breaks down the azimuthal symmetry $\mathbf{A}=(0,0, A(r, \theta))$. Hence, as long as a spherical basis is considered, a set of Debye scalar potentials $(\psi, \chi)$ for which $\mathbf{A}=\nabla \wedge(\mathbf{r} \psi)+\nabla \wedge \nabla \wedge(\mathbf{r} \chi)$ is used to solve the vectorial Helmholtz equation on $\mathbf{A}$. These potentials are solutions of the scalar Helmholtz equation so that $\psi$ and $\chi$ can also be decomposed in the spherical basis. Hence the incident, scattered and elastic waves in the sphere are readily described by four independent potentials decomposed in spherical modes. The boundary value problem yields the unknown scattering coefficients that are shown to be identical to the usual plane wave case [25].

At this stage, the total acoustic field can be computed and Eq.(15) yields the force exerted on the center of the sphere. It essentially depends on the material of the sphere and the fluid's properties through the scattering coefficients on one side, and on the nature of the incident field and the position of the sphere through the beam shape coefficients on the other. An interested reader can refer to [25] for further details on the derivation. Note that a similar result was obtained independently [61]. However, the generalized 
scattering problem was not addressed and the results restricted to rigid (no internal propagation) spheres.

The general treatment in Ref.[25] can be extended to account for various other physical effects. For example, the ECAH theory sets the necessary linear equations and introduces the vectorial treatment for elastic, viscous and thermal waves each of which can follow the same decomposition (Helmholtz and Debye) into a set of scalar potentials. These physical effects have shown to have a major influence on the radiation force on small particles compared to the wavelength $[62,63,64]$ and can be deduced from the ECAH theory $[65,66]$. Other extensions to the case of elastic shells $[67,68]$ were proposed in the context of the development of sonar detection. These models can find applications in radiation force calculations [69, 70].

\subsection{Computation of the beam shape coefficients}

In the context of GLMT theories, a major task resides in the accurate description of the incident beam and its position relative to the center of the scatterer. The beam shape coefficients fulfill this task and many techniques exist to obtain them. Their review is outside the scope of the present paper and the interested reader can refer to $[57,71]$. A numerically efficient implementation using rotation and addition theorems for spherical harmonics is available in optics [72] and was adapted to acoustics. Examples include helicoidal Bessel beams [25], focused axisymmetric and vortex beams [26].

\subsection{Acoustic radiation force in the long-wavelength limit}

When the spherical scatterer has a very small radius $a$ compared to the incident wavelength, the radiation force can be considerably simplified. On the one hand, only two vibrational modes of the sphere are excited. The first one is an isotropic monopolar expansion mode. It occurs when the compressibility of the scatterer differs from that of the fluid. The second one has a dipolar radiation pattern and arises from the back and forth oscillation of the sphere when a contrast of density between the two phases exists. In the small sphere limit, a Taylor expansion of the spherical Bessel functions involved in the two first scattering coefficients yields two different acoustic contrast factors [27]:

$$
\begin{aligned}
\alpha_{m} & =\alpha_{m}^{0} /\left(1+i \frac{k^{3}}{4 \pi} \alpha_{m}^{0}\right) \\
\alpha_{d} & =\alpha_{d}^{0} /\left(1-i \frac{k^{3}}{12 \pi} \alpha_{d}^{0}\right)
\end{aligned}
$$


where $k=\omega / c^{0}$ is the wave number in the liquid and

$$
\begin{aligned}
\alpha_{m}^{0} & =\frac{4}{3} \pi a^{3}\left(1-\frac{K^{0}}{K^{p}}\right) \\
\alpha_{d}^{0} & =4 \pi a^{3}\left(\frac{\rho^{p}-\rho^{0}}{2 \rho^{p}+\rho^{0}}\right) .
\end{aligned}
$$

$K^{0}=\rho\left(c^{0}\right)^{2}$ is the bulk elasticity of the material in the fluid and $K^{p}=$ $\rho^{p}\left(4 / 3 c_{t}^{2}-c_{l}^{2}\right)$ in the solid. The sphere's density, longitudinal and transverse wave speeds are noted $\rho^{p}, c_{l}$ and $c_{t}$ respectively.

On the other hand, the beam shape coefficients are formally scalar projections of the incident field on the spherical basis. In the same long wavelength limit, the beam shape coefficients can be written as linear combinations of the derivatives of the incident field taken at the center of the sphere [27]. The final expression of the force for a small elastic sphere in an inviscid fluid reads:

$$
\begin{aligned}
\mathbf{F}= & -\frac{1}{2}\left\{\Re\left(\alpha_{m}\right) \nabla\left(\frac{1}{2 \rho^{0}}\left(\frac{\left|P^{1}\right|}{c^{0}}\right)^{2}\right)-\Re\left(\alpha_{d}\right) \nabla\left(\frac{1}{2} \rho^{0}\left|\mathbf{v}^{1}\right|^{2}\right)\right. \\
& +\left(\frac{k}{c^{0}} \Im\left(\alpha_{m}\right)-\frac{k^{4}}{12 \pi c^{0}} \Re\left(\alpha_{m}\right) \Re\left(\alpha_{d}\right)\right) \Re\left(P^{1} \mathbf{v}^{1 *}\right) \\
& \left.+\rho^{0} \Im\left(\alpha_{d}\right) \Im\left(\left(\mathbf{v}^{1} \cdot \nabla\right) \mathbf{v}^{1 *}\right)\right\} .
\end{aligned}
$$

$\Re$ and $\Im$ denote the real and imaginary parts of these complex fields and * stands for a complex conjugations.

The first two terms in Eq.(34) stand for an acoustic gradient force. The real part of the monopolar scattering factor is associated with the gradient of the potential energy density of the field while the real part of the dipolar scattering factor is linked to the gradient of the kinetic energy density. It is a force proportional to the volume of the sphere $a^{3}$. Gorkov was probably the first to show that the radiation pressure of a standing wave field could be written as a gradient force [73]. His result is here recovered. The remaining term is called the scattering force and is associated to the imaginary parts of the scattering coefficients and a coupling between the monopolar and dipolar modes. Gorkov had also shown that the force exerted by a plane progressive wave was much weaker since there are no gradients in the fields' energy density. Indeed the imaginary part of the monopolar and dipolar contrast 
factors are multiplied by an additional factor $(k a)^{3} \ll 1$. Eq. 34 generalizes Gorkov's result to account for the scattering force of an arbitrary wavefield. A similar result was obtained elsewhere [54].

It is known that a very slight viscosity in the fluid suffices to drastically increase the magnitude of the scattering term of the force [62]. It is worth noting that the two acoustic contrast factors in Eq.(31) can me modified to account for the thermo-vicosity of the fluid or the visco-elasticity of the sphere $[64,66,65,70]$.

\subsection{Long-wavelength simplified expression in optics and acoustics}

The optical radiation force on a small dielectric sphere has a very similar expression [74],[75],[76]:

- There are also two modes but this time both are dipolar and related to the contrast in dielectric permittivity and magnetic permeability. Note that there is a single dipolar mode in acoustics because we assumed an elastic sphere in a fluid medium. The radiation pressure on a spherical inclusion in a solid would involve transverse incident waves.

- The real part of the scattering coefficient, the clausius-mossotti relation is identical to the acoustic dipolar scattering coefficients if specific mass is replaced by either the dielectric permittivity or the magnetic permeability.

- The scattering coefficients have a small imaginary part proportional to the square of the real part. This correction was recently introduced in optics [76].

- The force can be split in a gradient and a scattering force. In optics this was inferred independently of the correct expression of the scattering coefficients [75] and compared to the full theory provided by the GLMT.

- The gradient force is related to the energy density of the incident fields at the sphere center and the real part of the scattering coefficient.

- The scattering force is weaker and related to the imaginary part of the scattering coefficient. In both cases there is a term proportional to the mean of the Poynting vector. 
There are of course differences due to polarizations. In acoustics, the wave is scalar and longitudinally polarized in the liquid and have longitudinal and transverse components in the solid sphere. In optics the wave is transverse in both media.

\section{Acoustical tweezers}

Applying controlled forces without contact is appealing. It has many practical applications, both in optics and in acoustics. Optical tweezers can manipulate sub-micrometric objects with a nanometer resolution and forces in the pico-newton range. They have found a huge amount of applications from fundamental physics to material science and biophysics $[77,78,79,80$, 81]. Acoustic traps can considerably increase the size of the manipulated particles and the force that can be exerted. From the first acoustic levitation traps $[13,14]$ to the recent regain in interest in the context of acoustofluidics, acoustic traps have addressed a significant panel of new applications [15, $82,83]$. In this section we propose to review the recent demonstration of single-beam acoustical tweezers for which, in comparison to other traps, the development has been rather slow.

Unlike optical trapping of high-index particles, solid elastic materials are not transparent to ultrasound. The mechanical index mismatch is such that the preeminent mechanism at the fluid/solid boundary is backscattering instead of refraction. Moreover, considering a large sphere in the geometrical acoustics regime $(a \ll \lambda)$, not only the scattering force pushes the particle but, the refracted rays that in optics usually contribute to the restoring gradient component build up to an additional expelling force in acoustics. Restricting ourselves to much smaller spheres and avoiding as a first step the more complex Mie scattering regime $(a \leq \lambda)$, a quick analysis of Gorkovs theory (see Eq.(15)) shows that a particle that is denser and stiffer than the surrounding medium experiences a gradient force that points away from intensity maxima. Hence, whatever the size of the solid particle, acoustic beam traps generally exhibit an unstable behavior and the history of acoustic particle manipulation has almost always involved standing waves schemes.

Holding on to the single-beam concept, it was recently recognized that specific fields called acoustic vortices could act as stable lateral [84] and threedimensional traps [26]. The lateral trapping of vortex type beams was confirmed experimentally in a planar configuration creating 2D annular Bessel function shaped traps [85]. Using a three-dimensional theory for acoustic 
forces [25] is was possible to compute the axial trapping force of tightly focused vortex beams [26]. It was concluded that a focused vortex beam of topological charge $m=1$ could generate a negative axial gradient force to stably trap elastic particles in three dimensions. In fact, using sufficiently small spheres $(a \leq 0.15 \lambda)$, the scattering force is greatly diminished on the axis of a vortex beam meanwhile the gradient component points this time towards the focus. Note that other types of beams were proposed in [26] to enhance the axial trapping efficiency similar to what is referred to as "bottle beams" in optics $[86,87]$.

The research field of structured wavefronts is again intimately related between optics and acoustics. Though the seminal paper published by Nye and Berry in 1974 first introduced the phenomenon of phase singularities within ultrasonic wave trains [88], the optics community rapidly pushed the concept forward towards a whole deal of fundamental studies and applications $[89,90,91]$. Remarkably, a route to directly create a beam carrying a screw phase dislocation was first theoretically proposed in acoustics [92] at the time it was recognized that $\mathrm{TEM}_{01}^{*}$ laser modes could be generated $[91,93]$. Experimentally demonstrated by Hefner and Marston [94]. Studies of their linear and non-linear behavior include the establishment of a law of conservation of their topological charge and pseudo-angular momentum [94, 95, 96], vortex parametric interaction [97], azimuthal shock waves [98] and their super-oscillation properties applied to sub-wavelength imaging [99]. An example of a synthesized vortex beam is given in Fig.3. The ultrasonic field is generated by a 128 element piezoelectric array in a water tank using the inverse filter technique [95]. As for its optical counterpart, the energy is focused to a ring in the focal plane (panel a)). Note that a hydrophone delivers a direct measurement of the spiraling phase structure while in optics the vortex beam generally has to interfere with a plane wave. It is noteworthy that a high numerical aperture acoustic lens was designed to focus the beam to a ring of diameter comparable to $\lambda$. The region of undefined phase is a line in three dimensions. Consequently, the entire propagation axis defines a silent zone (see Fig.3c)).

The control achieved in creating tightly focused vortex beams led us to experimentally demonstrate the existence of a negative gradient force and in essence observe the first single-beam gradient trap for elastic particles with an ultrasonic beam [27]. In the first configuration a vortex was fired horizontally in the water tank while a polystyrene particle was approached near the focal region by an auxiliary tee. Figure 4 shows a photograph of a $400 \mu \mathrm{m}$ size 

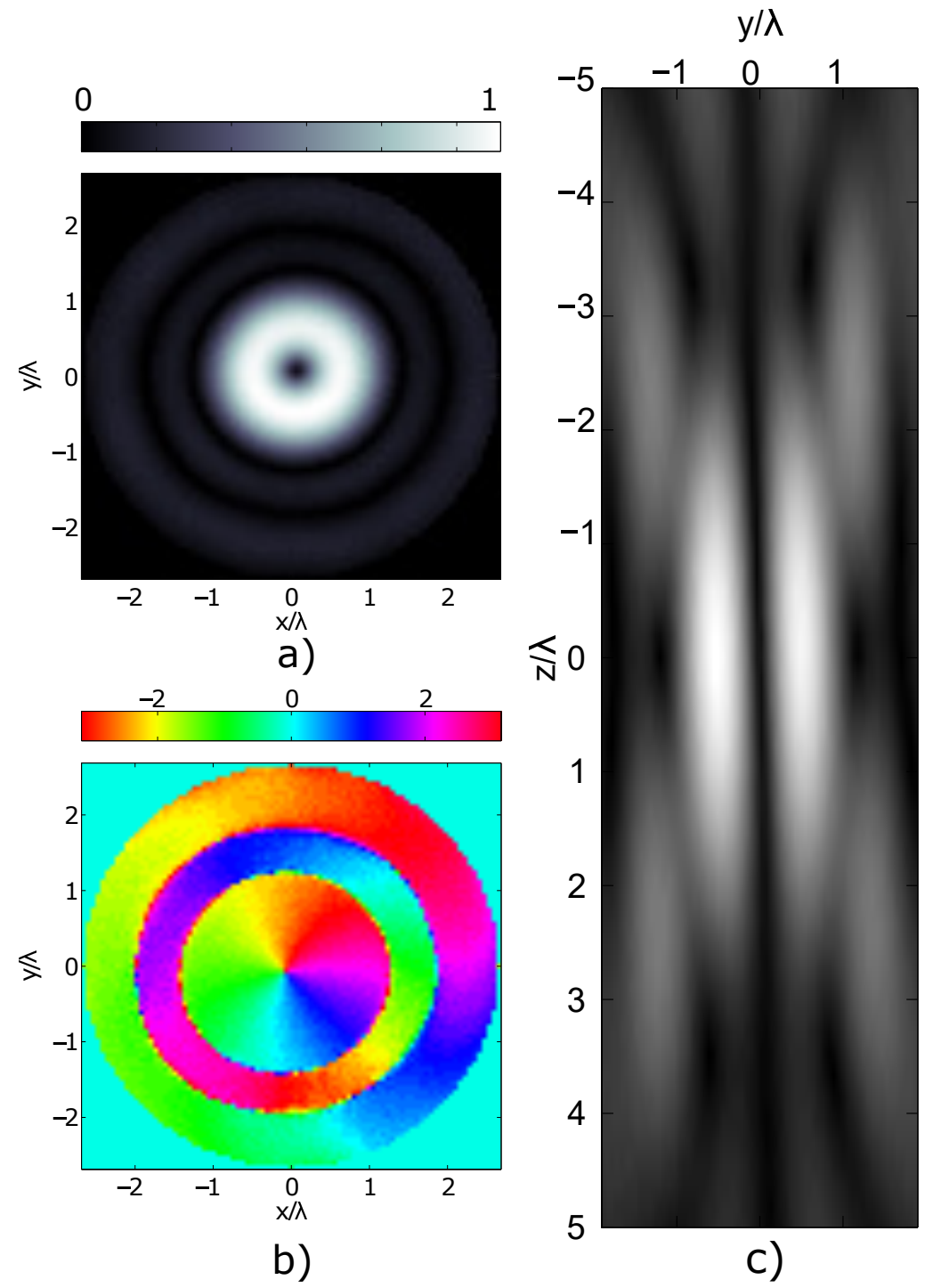

b)

a)

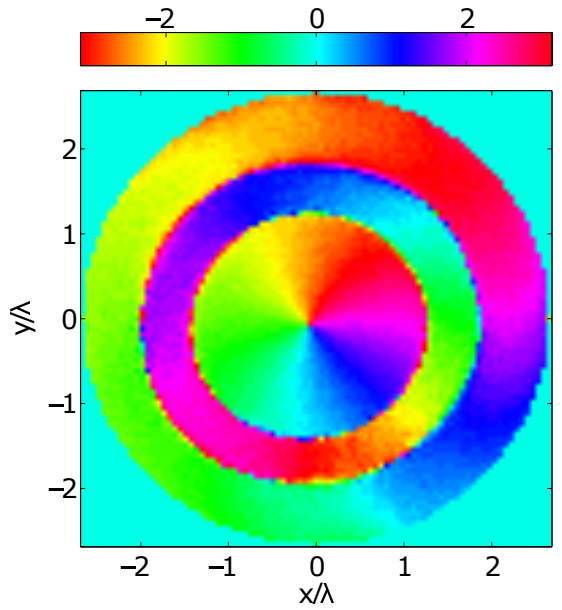

c)

Figure 3: Example of a synthesized vortex beam of topological charge $m=1$. a) and b), normalized intensity and phase (rad.) in the focal plane respectively. c), normalized intensity along the propagation axis. Adapted from [65]. 


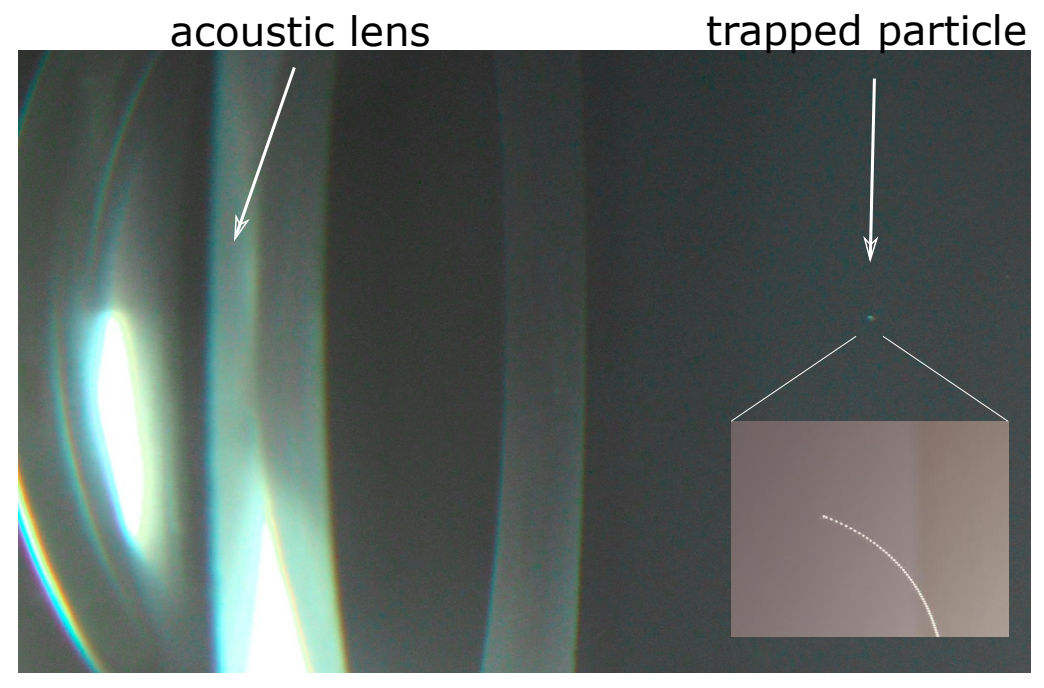

Figure 4: Photograph of a trapped polystyrene particle in a horizontal acoustical tweezers setup. A zoomed range of the particle's release is displaced with an overlap of images. The particle's trajectory shows the effect of gravity and acoustic streaming.

polystyrene sphere trapped in the focus of the helicoidal field. The particle levitates approximately $30 \mathrm{~mm}$ away from the outer face of the lens as long as the vortex is emitted. When the source is turned off, an overlapped image stack of the release of the sphere is shown. It can be seen from the trajectory that at this scale that gravity plays a major role and remark the significant effect of acoustic streaming pushing the particle away from the focus.

It was decided to demonstrate that the negative gradient force was so large that it dominated the axial stability in a vertical configuration. The experimental setup described in [27] was used to lift and trap buoyant particles against their weight and the pushing force exerted by the streaming flow's drag. Figure 5 is a photograph of a $340 \mu \mathrm{m}$ polystyrene bead trapped and levitated beneath the focus of the vortex beam. The bead was initially lying on an acoustically transparent polyethylene film. By precisely aiming the beam, the tweezers can accurately select the particle to be trapped. Other particles can be slightly affected but will not collect in the the focal volume. 


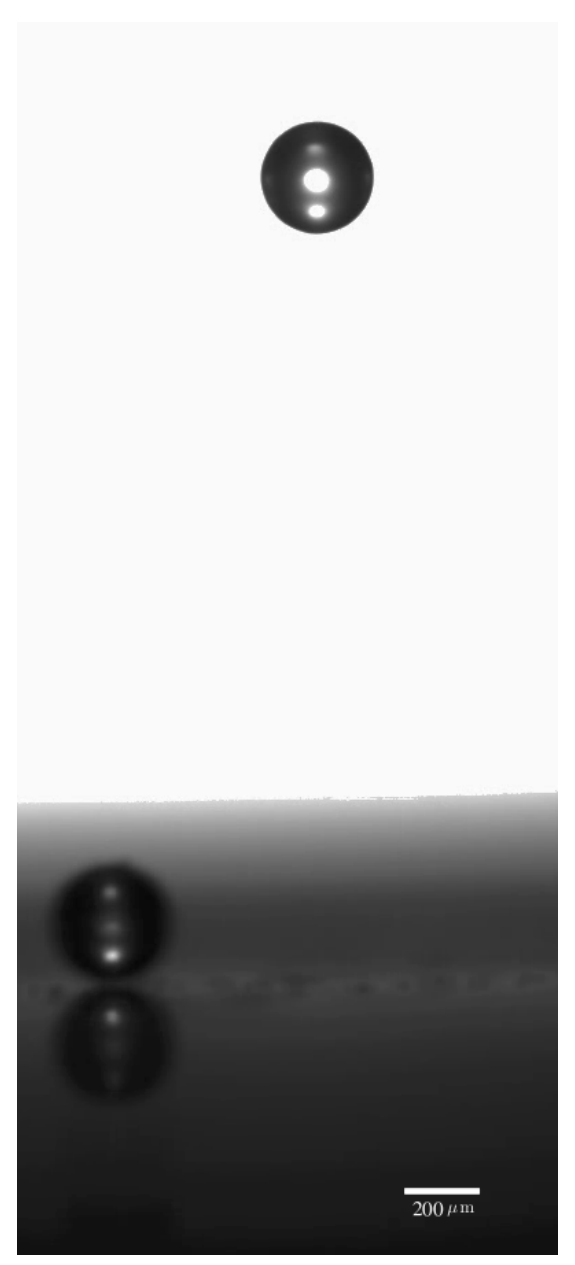

Figure 5: Photograph of a $340 \mu \mathrm{m}$ diameter polystyrene sphere trapped in vertical acoustical tweezers. The trapped particle was selected among others that were lying on a thin polyethylene film. Adapted from [27] 


\section{Conclusion}

The radiation pressures exerted by sound or light have many similar features. This was shown with the expression of the stress tensor and the relation with pseudo-momentum. Not only these second order effects are similar but also when the linear problem of a spherical scatterer interacting with an incident beam is considered. The Generalized Lorenz-Mie theory has therefore recently found an adaptation to acoustic scattering and force calculations. Regarding the force in the long wavelength limit, it was shown that a beam exerted both a gradient and scattering force in acoustics in a similar fashion to the force exerted by an optical beam on a small dielectric dipole. The possibility to design manipulation traps for small particles is another appealing and common characteristic. Among all kinds of traps, optical tweezers stand out by their simplicity, accurate localized actuation and great dexterity. Quoting Ashkin [20]: "The single-beam gradient force trap is conceptually and practically one of the simplest radiation pressure traps". A feature which most certainly explains their wide application in various scientific fields. The attention of the acoustic community has essentially been turned towards standing wave traps where Gorkov's theory has found a sound application. They have addressed a large panel of new applications in the context of acoustical levitation or acoustofluidics [15, 82, 83]. A recent review offers an extensive survey of various developed devices [100]. The development of single-beam acoustical tweezers had been impaired by two main difficulties: the lack of a complete theoretical model able to predict the force exerted by acoustic beams and the capacity to synthesize acoustic beams with complex wavefronts as focused acoustical vortices. The complex wavefield synthesis method with a large array of piezo-electric transducers was adapted from previous studies on acoustical vortices [95, 96].

The thermal damage, or "opticution", limit optical tweezers to applications requiring very weak forces and to manipulate very small particles from atoms to molecules. Ultrasound are proven to innocuous for living cells and propagate without significant attenuation in many materials. This feature combined with the much larger forces applied at equivalent energy flux should give to single-beam acoustical tweezers an extended range of manipulation capacities for new applications in material science, fluidics and biophysics.

[1] J. C. Maxwell, Art. 314. medium in which small spheres are uniformly disseminated, chapter ix. conduction through heterogeneous media, A treatise on electricity and magnetism 1. 
[2] P. Lebedev, Experimental examination of light pressure, Annalen der physik 6 (1901) 433.

[3] E. F. Nichols, G. F. Hull, The pressure due to radiation, in: Daedalus, Vol. 38, 1903, pp. 559-599.

[4] L. Rayleigh, Phil. Mag. 3 (1902) 338.

[5] L. Rayleigh, Phil. Mag. 10 (1905) 364.

[6] V. Dvorak, On acoustic repulsion, Am. J. Sci. 16 (1878) 22-29.

[7] W. Altberg, ber die druckkrfte der schallwellen und die absolute messung der schallintensitt, Annalen der Physik 316 (6) (1903) 405-420.

[8] C. Eckart, Vortices and streams caused by sound waves 73 (1) (1948) 68-76.

[9] R. Peirls, Momentum and pseudomomentum of light and sound, Proc. Intl. School Phys. "Enrico Fermi", Elsevier Science Ltd, 1985, pp. 237255.

[10] M. McIntyre, On the "wave momentum" myth, J. Fluid. Mech. 106 (1981) 331-347.

[11] L. Brillouin, Tensors in mechanics and elasticity, Academic Press, New York, 1964.

[12] A. Eller, Force on a bubble in a standing acoustic wave, J. Acoust. Soc. Am. 43 (1) (1968) 170-171.

[13] E. Trinh, Compact acoustic levitation device for studies in fluid dynamics and material science in the laboratory and microgravity, Rev. Sci. Instrum. 56 (11) (1985) 2059-2065.

[14] R. E. Apfel, Acoustic levitation for studying liquids and biological materials, J. Acoust. Soc. Am. 70 (2) (1981) 636-639.

[15] T. Laurell, F. Petersson, A. Nilsson, Chip integrated strategies for acoustic separation and manipulation of cells and particles, Chem. Soc. Rev. 36 (3) (2007) 492-506. 
[16] P. B. Muller, M. Rossi, Á. Marín, R. Barnkob, P. Augustsson, T. Laurell, C. J. Kaehler, H. Bruus, Ultrasound-induced acoustophoretic motion of microparticles in three dimensions, Phys. Rev. E 88 (2) (2013) 023006 .

[17] F. Guo, P. Li, J. B. French, Z. Mao, H. Zhao, S. Li, N. Nama, J. R. Fick, S. J. Benkovic, T. J. Huang, Controlling cell-cell interactions using surface acoustic waves, Proc. Natl. Acad. Sci. USA 112 (1) (2015) $43-48$.

[18] C. R. Courtney, C. E. Demore, H. Wu, A. Grinenko, P. D. Wilcox, S. Cochran, B. W. Drinkwater, Independent trapping and manipulation of microparticles using dexterous acoustic tweezers, App. Phys. Lett. 104 (15) (2014) 154103.

[19] A. Ashkin, Acceleration and trapping of particles by radiation pressure, Phys. Rev. Lett. 24 (4) (1970) 156-159.

[20] A. Ashkin, J. Dziedzic, J. Bjorkholm, S. Chu, Observation of a singlebeam gradient force optical trap for dielectric particles, Optics letters 11 (5) (1986) 288-290.

[21] J. Wu, G. Du, Acoustic radiation force on a small compressible sphere in a focused beam, J. Acoust. Soc. Am. 87 (3) (1990) 997-1003.

[22] J. Wu, Acoustical tweezers, J. Acoust. Soc. Am. 89 (5) (1991) 21402143.

[23] A. Ashkin, How it all began, Nature Photonics 5 (2011) 316-317.

[24] J. Lee, K. Shung, Radiation forces exerted on arbitrarily located sphere by acoustic tweezer, J. Acoust. Soc. Am. 120 (2) (2006) 1084-1094.

[25] D. Baresch, J.-L. Thomas, R. Marchiano, Three-dimensional acoustic radiation force on an arbitrarily located elastic sphere, J. Acoust. Soc. Am. 133 (1) (2013) 25-36.

[26] D. Baresch, J.-L. Thomas, R. Marchiano, Spherical vortex beams of high radial degree for enhanced single-beam tweezers, J. Appl. Phys. 113 (18) (2013) 184901. 
[27] D. Baresch, J.-L. Thomas, R. Marchiano, Observation of a single-beam gradient force acoustical trap for elastic particles: acoustical tweezers, Phys. review lett. 116 (2) (2016) 024301.

[28] A. Marzo, S. A. Seah, B. W. Drinkwater, D. R. Sahoo, B. Long, S. Subramanian, Holographic acoustic elements for manipulation of levitated objects, Nat. Commun. 6 (2015) 8661.

[29] J. Jackson, Classical Electrodynamics, John Wiley and Sons Inc., New York, 1962, Ch. 6.

[30] K. Beissner, The acoustic radiation force in lossless fluids in eulerian and lagrangian coordinates, J. Acoust. Soc. Am. 103 (5) (1998) 23212332 .

[31] E. J. Post, Radiation pressure and dispersion, J. Acoust. Soc. Am. 25 (1) (1953) 55-60.

[32] K. Beissner, Two concepts of acoustic radiation pressure, J. Acoust. Soc. Am. 79 (5) (1986) 1610-1612.

[33] R. Beyer, Radiation pressure-the history of a mislabeled tensor, J. Acous. Soc. Am. 63 (4) (1978) 1025-1030.

[34] P. Biquard, Rev. Acoust. 1 (1932) 93-109.

[35] P. Biquard, Rev. Acoust. 2 (1933) 315-335.

[36] B. Chu, R. E. Apfel, Acoustic radiation pressure produced by a beam of sound, J. Acous. Soc. Am. 72 (6) (1982) 1673-1687.

[37] J. Faran, Sound scattering by solid cylinders and spheres, J. Acoust. Soc. Am. 23 (1951) 405-418.

[38] P. Epstein, R. Carhart, The absorption of sound in suspensions and emulsions. i. water fog in air., J. Acoust. Soc. Am. 25 (3) (1953) 553565 .

[39] J. Allegra, S. Hawley, Attenuation of sound in suspensions and emulsions: Theory and experiments, J. Acoust. Soc. Am. 51 (5) (1971) 1545-1564. 
[40] P. M. Morse, Vibration and sound, Vol. 2, McGraw-Hill New York, 1948.

[41] K. Yosioka, Y. Kawasima, Acoustic radiation pressure on a compressible sphere, Acustica 5 (3) (1955) 167-173.

[42] T. Hasegawa, K. Yosioka, Acoustic radiation force on a solid elastic sphere, J. Acoust. Soc. Am. 46 (5) (1969) 1139-1143.

[43] N. A. Logan, Early history of the mie solution, J. Opt. Soc. Am. 52 (3) (1962) 342-343.

[44] N. A. Logan, Survey of some early studies of the scattering of plane waves by a sphere, Proc. IEEE 53 (8) (1965) 773-785.

[45] N. Einspruch, E. Witterholt, R. Truell, Scattering of a plane transverse wave by a spherical obstacle in an elastic medium, J. Appl. Phys. 31 (5) (1960) 806-818.

[46] G. C. Gaunard, H. Überall, Theory of resonant scattering from spherical cavities in elastic and viscoelastic media, J. Acoust. Soc. Am. 63 (6) (1978) 1699-1712.

[47] T. Nieminen, H. Rubinsztein-Dunlop, N. Heckenberg, Calculation of the t-matrix: general considerations and application of the pointmatching method, J. Quant. Spect. and Rad. Transf. 7980 (2003) 1019 -1029 .

[48] P. Waterman, New formulation of acoustic scattering, J. Acoust. Soc. Am. 45 (6) (1969) 1417-1429.

[49] P. Waterman, Matrix theory of elastic wave scattering, J. Acoust. Soc. Am. 60 (3) (1976) 567-580.

[50] X. Chen, R. Apfel, Radiation force on a spherical object in the field of a focused cylindrical transducer, J. Acoust. Soc. Am. 101 (5) (1996) $2443-2447$.

[51] P. L. Marston, Axial radiation force of a bessel beam on a sphere and direction reversal of the force, J. Acoust. Soc. Am. 120 (6) (2006) 3518. 
[52] P. L. Marston, Scattering of a bessel beam by a sphere: Ii helicoidal case and spherical shell example, J. Acoust. Soc. Am. 124 (5) (2008) 2905-2910.

[53] P. L. Marston, Radiation force of a helicoidal bessel beam on a sphere, J. Acoust. Soc. Am. 120 (2009) 3539-3547.

[54] O. A. Sapozhnikov, M. R. Bailey, Radiation force of an arbitrary acoustic beam on an elastic sphere in a fluid, J. Acoust. Soc. Am. 133 (2) (2013) 661-676.

[55] B. Maheu, G. Gouesbet, G. Gréhan, A concise presentation of the generalized lorenz-mie theory for arbitrary location of the scatterer in an arbitrary incident profile, J. Opt. 19 (2) (1987) 59-67.

[56] G. Gouesbet, B. Maheu, G. Gréhan, Light scattering from a sphere arbitrarily located in a gaussian beam, using a bromwich formulation, JOSA A 5 (9) (1988) 1427-1443.

[57] G. Gouesbet, J. Lock, G. Gréhan, Generalized lorenzmie theories and description of electromagnetic arbitrary shaped beams : Localized approximations and localized beam models, a review, J. Quant. Spect. and Rad. Transf. 112 (2010) 1-27.

[58] J. P. Barton, D. R. Alexander, S. A. Schaub, Internal and near-surface electromagnetic fields for a spherical particle irradiated by a focused laser beam, J. Appl. Phys. 64 (4) (1988) 1632-1639.

[59] K. Ren, G. Gréhan, G. Gouesbet, Radiation pressure forces exerted on a particle arbitrarily located in a gaussian beam by using the generalized lorenz-mie theory, and associated resonance effects, Optics Comm. 108 (1994) 343-354.

[60] J. P. Barton, D. R. Alexander, S. A. Schaub, Theoretical determination of net radiation force and torque for a spherical particle illuminated by a focused laser beam, J. Appl. Phys. 66 (10) 4594-4602.

[61] G. T. Silva, An expression for the radiation force exerted by an acoustic beam with arbitrary wavefront, J. Acoust. Soc. Am. 130 (6) (2011) $3541-3544$. 
[62] S. Danilov, M. Mironov, Mean force on a small sphere in a sound field in a viscous fluid, J. Acoust. Soc. Am. 107 (1) (2000) 143-153.

[63] A. A. Doinikov, Acoustic radiation pressure on a rigid sphere in a viscous fluid, Proc Royal Soc. London. A. 447 (1994) 447-466.

[64] M. Settnes, H. Bruus, Physical Review E 85 (1) (2012) 016327.

[65] D. Baresch, Pince acoustique: piégeage et manipulation d'un objet par pression de radiation d'une onde progressive, Ph.D. thesis, Paris 6 (2014).

[66] J. T. Karlsen, H. Bruus, Forces acting on a small particle in an acoustical field in a thermoviscous fluid, Phys. Rev. E 92 (2015) 043010.

[67] V. Ayres, G. C. Gaunaurd, Acoustic Resonance Scattering by Viscoelastic Objects, J. Acoust. Soc. Am. 81 (2).

[68] M. C. Junger, Sound scattering by thin elastic shells, The Journal of the Acoustical Society of America 24 (4) (1952) 366-373.

[69] T. Hasegawa, Y. Hino, A. Annou, H. Noda, M. Kato, N. Inoue, Acoustic radiation pressure acting on spherical and cylindrical shells, The Journal of the Acoustical Society of America 93 (1) (1993) 154-161.

[70] J. P. Leão Neto, J. H. Lopes, G. T. Silva, Core-shell particles that are unresponsive to acoustic radiation force, Phys. Rev. Applied 6 (2016) 024025 .

[71] T. Nieminen, H. Rubinsztein-Dunlop, N. Heckenberg, Multipole expansion of strongly focussed laser beams, J. Quant. Spect. and Rad. Transf. 79 (2003) 1005-1017.

[72] T. Nieminen, V. Loke, A. Stilgoe, G. Knner, A. Branczyk, N. Heckenberg, Optical tweezers computational toolbox, J. Opt. A : Pure Appl. Opt. 9 (2007) 196-203.

[73] L. Gor'kov, On the forces acting on a small particle in an acoustic field in an ideal fluid, Sov. Phys. Dokl 6 (1962) 773-775.

[74] T. A. Nieminen, G. Knöner, N. Heckenberg, H. Rubinsztein-Dunlop, Physics of optical tweezers, Methods Cell Biol. 82 (2007) 207-236. 
[75] Y. Harada, T. Asakura, Radiation forces on a dielectric sphere in the rayleigh scattering regime, Optics Comm. 124 (1996) 529-541.

[76] P. Chaumet, M. Nieto-Vesperinas, Coupled dipole method determination of the electromagnetic force on a particle over a flat dielectric substrate, Phys. Rev. B 61 (20) (2000) 14119-14127.

[77] A. Ashkin, J. Dziedzic, T. Yamane, Optical trapping and manipulation of single cells using infrared laser beams, Nature 330 (6150) (1987) 769771.

[78] D. G. Grier, A revolution in optical manipulation, Nature 424 (6950) (2003) 810-816.

[79] K. Dholakia, T. Čižmár, Shaping the future of manipulation, Nature Photonics 5 (6) (2011) 335-342.

[80] A. Aspect, E. Arimondo, R. Kaiser, N. Vansteenkiste, C. CohenTannoudji, Laser cooling below the one-photon recoil energy by velocity-selective coherent population trapping, Phys. Rev. Lett. 61 (7) (1988) 826.

[81] M. Padgett, S. Barnett, R. Loudon, The angular momentum of light inside a dielectric, J. Mod. Opt 50 (10) (2003) 1555-1562.

[82] J. Friend, L. Y. Yeo, Microscale acoustofluidics: Microfluidics driven via acoustics and ultrasonics, Reviews of Modern Physics 83 (2) (2011) 647.

[83] M. Evander, J. Nilsson, Acoustofluidics 20: applications in acoustic trapping, Lab on a chip 12 (22) (2012) 4667-4676.

[84] S. Kang, C. Yeh, Potential-well model in acoustic tweezers, IEEE Ultrasonics 57 (6) (2010) 1451-1459.

[85] C. R. Courtney, B. W. Drinkwater, C. E. Demore, S. Cochran, A. Grinenko, P. Wilcox, Dexterous manipulation of microparticles using bessel-function acoustic pressure fields, App. Phys. Lett. 102 (12) (2013) 123508. 
[86] J. Arlt, M. J. Padgett, Generation of a beam with a dark focus surrounded by regions of higher intensity:the optical bottle beam, Opt. Lett. 25 (4) (2000) 191-193.

[87] C.-H. Chen, P.-T. Tai, W.-F. Hsieh, Bottle beam from a bare laser for single-beam trapping, Appl. Opt. 43 (32) (2004) 6001-6006.

[88] J. Nye, M. Berry, Dislocations in wave trains, Proc. R. Soc. London 336 (1974) 165-190.

[89] M. Soskin, M. Vasnetsov, Singular optics, Progress in optics 42 (2001) 219-276.

[90] G. Gibson, J. Courtial, M. J. Padgett, M. Vasnetsov, V. Pasko, S. M. Barnett, S. Franke-Arnold, Free-space information transfer using light beams carrying orbital angular momentum, Optics Express 12 (22) (2004) 5448-5456.

[91] M. R. Dennis, K. O'Holleran, M. J. Padgett, Singular optics: optical vortices and polarization singularities, Progress in Optics 53 (2009) $293-363$.

[92] C. Cain, S. Umemura, Concentric-ring and sector-vortex phased-array applicators for ultrasound hyperthermia, IEEE Transactions on Microwave Theory and Techniques 34 (5) (1986) 542-551.

[93] C. Tamm, C. O. Weiss, Bistability and optical switching of spatial patterns in a laser, J. Opt. Soc. Am. B 7 (6) (1990) 1034-1038.

[94] B. T. Hefner, P. L. Marston, An acoustical helicoidal wave transducer with applications for the alignment of ultrasonic and underwater systems, J. Acous. Soc. Am. 106 (6) (1999) 3313-3316.

[95] J.-L. Thomas, R. Marchiano, Pseudo angular momentum and topological charge conservation for nonlinear acoustical vortices, Phys. Rev. Lett. 91 (24) (2003) 1-4.

[96] R. Marchiano, J.-L. Thomas, Synthesis and analysis of linear and nonlinear acoustical vortices, Phys. Rev. E. 71 (2005) 1-11.

[97] R. Marchiano, J.-L. Thomas, Doing arithmetic with nonlinear acoustic vortices, Phys. Rev. Lett. 101 (2008) 1-4. 
841

842

843

844

845

846

847

848

[98] T. Brunet, J.-L. Thomas, R. Marchiano, F. Coulouvrat, Experimental observation of azimuthal shock waves on nonlinear acoustical vortices, New J. Phys. 11 (2009) 013002.

[99] T. Brunet, J.-L. Thomas, R. Marchiano, Transverse shift of helical beams and subdiffraction imaging, Phys. Rev. Lett. 105 (3) (2010) 034301.

[100] B. W. Drinkwater, Dynamic-field devices for the ultrasonic manipulation of microparticles, Lab on a Chip 16 (2016) 2360-2375. 